\title{
OPEN The effect of pre-resection obesity on post-resection body composition after $75 \%$ small bowel resection in rats
}

\begin{abstract}
Neesha S. Patel ${ }^{1,2}$, Ujwal R. Yanala ${ }^{1,2}$, Shruthishree Aravind ${ }^{1,2}$, Roger D. Reidelberger ${ }^{2,3}$, Jon S. Thompson ${ }^{1} \&$ Mark A. Carlson ${ }^{1,2,4,5 \bowtie}$

In patients with short bowel syndrome, an elevated pre-resection Body Mass Index may be protective of post-resection body composition. We hypothesized that rats with diet-induced obesity would lose less lean body mass after undergoing massive small bowel resection compared to non-obese rats. Rats (CD IGS; age = $2 \mathrm{mo} ; \mathrm{N}=80$ ) were randomly assigned to either a high-fat (obese rats) or a low-fat diet (non-obese rats), and fed ad lib for six months. Each diet group then was randomized to either underwent a 75\% distal small bowel resection (massive resection) or small bowel transection with re-anastomosis (sham resection). All rats then were fed ad lib with an intermediate-fat diet ( $25 \%$ of total calories) for two months. Body weight and quantitative magnetic resonance-determined body composition were monitored. Preoperative body weight was $884 \pm 95$ versus $741 \pm 75 \mathrm{~g}$, and preoperative percent body fat was $35.8 \pm 3.9$ versus $24.9 \pm 4.6 \%$; high-fat vs. low fat diet, respectively $(p<0.0001)$; preoperative diet type had no effect on lean mass. Regarding total body weight, massive resection produced an $18 \%$ versus $5 \%$ decrease in high-fat versus low-fat rats respectively, while sham resection produced a $2 \%$ decrease vs. a $7 \%$ increase, respectively $(p<0.0001$, preoperative vs. necropsy data). Sham resection had no effect on lean mass; after massive resection, both high-fat and low-fat rats lost lean mass, but these changes were not different between the latter two rat groups. The high-fat diet and low-fat diet induced obesity and marginal obesity, respectively. The massive resection produced greater weight loss in high-fat rats compared to low-fat rats. The type of dietary preconditioning had no effect on lean mass loss after massive resection. A protective effect of preexisting obesity on lean mass after massive intestinal resection was not demonstrated.
\end{abstract}

The prevalence of short bowel syndrome (SBS) in United States is in the range of 30-45 per million ${ }^{1,2}$, but SBS may be under-reported due to lack of a national registry or a database and variation in definition. The incidence often is estimated from home parenteral nutrition registries ${ }^{2}$. A Swedish SBS registry estimated an SBS prevalence of 44 per million in that country ${ }^{3}$. Approximately $15 \%$ of adults who undergo massive intestinal resection will suffer from SBS ${ }^{4,5}$. A process of structural and functional adaptation occurs in SBS in which the intestinal remnant hypertrophies and dilates, thereby increasing the absorptive capacity of the intestines ${ }^{6}$. In addition, a distinct process called adaptive hyperphagia has been described in patients with SBS, wherein the dietary intake is markedly increased as a compensatory mechanism for reduced absorptive capacity ${ }^{7}$.

Clinical studies have suggested that the pre-resection body mass index (BMI) influences post-resection BMI in patients with $\mathrm{SBS}^{8,9}$. Obesity may affect the intestinal adaptive response, energy expenditure, body composition and adaptive hyperphagia after intestinal resection ${ }^{9}$. In order to determine whether pre-existing obesity would be protective of body weight and other nutritional endpoints in experimental SBS, we previously ${ }^{10}$ performed a study on the effect of a $50 \%$ small intestinal resection on nutritional endpoints in rats with and without dietinduced obesity. The obese rats may have retained more lean body mass after resection that non-obese rats,

${ }^{1}$ Department of Surgery, University of Nebraska Medical Center, 983280 Nebraska Medical Center, Omaha, NE 68198-3280, USA. 'Surgery Department, VA Medical Center, 4101 Woolworth Avenue, Omaha, NE 68105, USA. ${ }^{3}$ Department of Biomedical Sciences, Creighton University, Omaha, NE 68178, USA. ${ }^{4}$ Center for Advanced Surgical Technology, University of Nebraska Medical Center, 986245 Nebraska Medical Center, Omaha, NE 68198-6245, USA. ${ }^{5}$ Department of Genetics, Cell Biology and Anatomy, University of Nebraska Medical Center, 985805 Nebraska Medical Center, Omaha, NE 68198-5805, USA.『email: macarlso@unmc.edu 


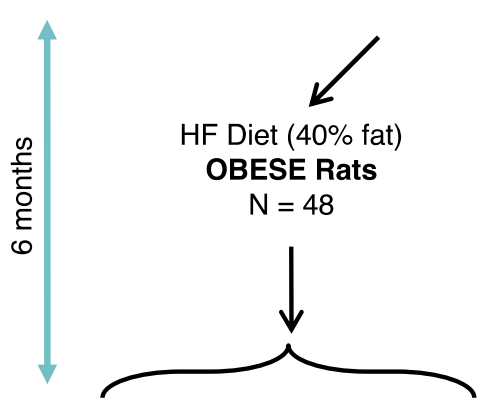
(1) Sham

(2) Massive

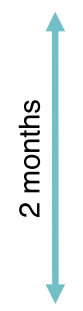

Rats (CD IGS, male, $3 \mathrm{mo}$ )
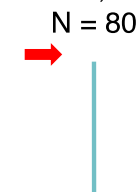

Dietary

Induction

Phase

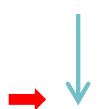

OPERATION

(3) Sham

$\mathrm{N}=15$

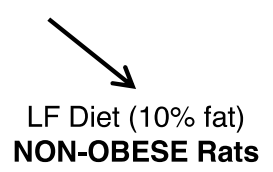

$\mathrm{N}=32$
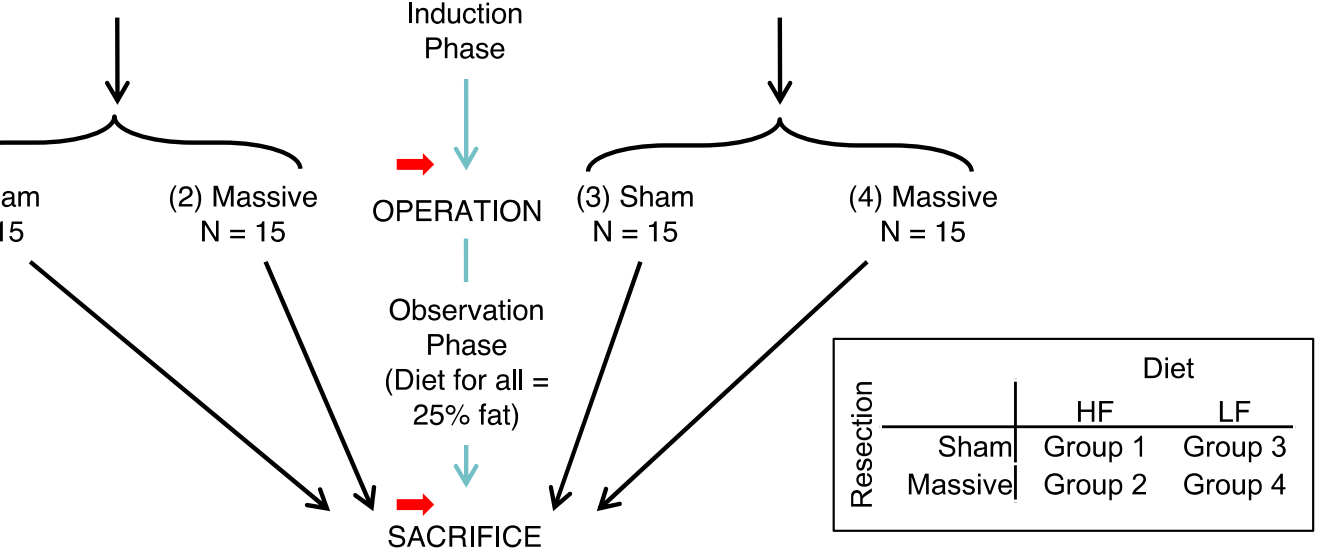

Figure 1. Experimental Design. $\mathrm{HF}=$ High-Fat; $\mathrm{LF}=\mathrm{Low}-\mathrm{Fat} ;$ Sham $=$ sham resection; Massive $=75 \%$ distal small intestinal resection. Red arrow = body composition measurement. Inset: $2 \times 2$ matrix of experimental groups.

but the difference was small and not statistically significant ${ }^{10}$. Herein we report a repeat of the above rat study, but this time using a greater intestinal resection ( $75 \%$ of the small bowel) and a longer postoperative follow-up period (two months, as opposed to the previous one-month period). The rationale for this repeat study was to follow up on the inconclusive results of the previous study ${ }^{10}$, using a more severe model of SBS to determine whether rats with diet-induced obesity would have greater preservation of lean mass after massive resection than non-obese rats.

\section{Materials and methods}

Animal welfare. This study was performed with approval from the Institutional Animal Care and Use Committees of the VA Nebraska-Western Iowa Health Care System (protocol number 00992) and of the University of Nebraska Medical Center (Protocol Number 14-082-09-ET), and in accordance with the recommendations in the NIH Guide for the Care and Use of Laboratory Animals (8th ed.) ${ }^{11}$. Procedures were performed and subjects housed in animal facilities approved by the Association for Assessment and Accreditation of Laboratory Animal Care International (AAALAC; www.aaalac.org). Animals were housed in pairs, except for a period of single housing for 7 days after surgery. Surgical procedures were performed under isoflurane anesthesia, and all efforts were made to minimize pain and distress. Buprenorphine SR $(0.1 \mathrm{mg} / \mathrm{kg}$ injection $)$ was given as needed or pain. Any subject in distress, not eating, not moving, or with obvious wound infection was euthanized. Euthanasia was performed in accordance with the AVMA Guidelines ${ }^{12}$. The ARRIVE 2.0 Guidelines (Animal Research: Reporting of In Vivo Experiments ${ }^{13,14}$ ) Checklist is shown in Fig. S1.

Animal subjects and determination of subject numbers. CD IGS (International Genetic Standard) rats (all males; age 3 mo upon arrival) were purchased from Charles River Laboratories International, Inc. (The minimum number of rats $(\mathrm{N}=12)$ utilized for each experimental group (four groups total; see Fig. 1) was determined with a statistical power analysis using $\Delta / \sigma$ (=Cohen's $d$, in which $\Delta$ is the desired difference in means as set by the observer, and $\sigma$ is the estimated standard deviation $)=1.50$, false positive rate $(\alpha)=0.05$, false negative rate $(\beta)=0.2$, and power $(1-\beta)=0.8$. With $N=12$ rat/group and $\alpha$ and $\beta$ set as above, a $20 \%$ difference among means could be detected with a standard deviation equal to $13 \%$ of the mean. The primary endpoint in this study was lean body mass. The actual number of subjects per group at the beginning of the dietary induction phase in Fig. 1 was increased to 15 . The justification for this increased subject number was in case of subject loss secondary to illness or perioperative mortality.

Experimental design overview. The experimental design (Fig. 1) was adapted from our previous study ${ }^{10}$. Rats were assigned to either a High-Fat (HF) or a Low-Fat (LF) diet for 120 days, and then underwent either a $75 \%$ distal enterectomy ("massive resection") or a transection of the distal ileum with anastomosis ("sham resection"). This design yielded a $2 \times 2$ matrix of experimental groups (Fig. 1). Postoperatively all animals were maintained on an Intermediate-Fat (IF) diet for 60 days, and then euthanized. Body composition was measured prior to dietary induction, prior to operation, and prior to sacrifice (Fig. 1). 


\begin{tabular}{|l|l|l|l|}
\hline Quantity & High-fat diet $^{*}$ & Low-fat diet $^{\dagger}$ & Intermediate-fat diet $^{*}$ \\
\hline Energy density (kcal/g) & 4.73 & 3.85 & 4.73 \\
\hline Protein kcal (\%) & 20 & 20 & 24 \\
\hline Carbohydrate kcal (\%) & 35 & 70 & 41 \\
\hline Fat kcal (\%) & 45 & 10 & 25 \\
\hline
\end{tabular}

Table 1. Dietary formulations. High-fat $\operatorname{diet}^{*}=\mathrm{D} 12451$; low fat $\operatorname{diet}^{\dagger}=\mathrm{D} 12450 \mathrm{~B}$ (both from OpenSource Diets); intermediate fat diet ${ }^{*}=$ LabDiet 5001 Rodent Diet (PMI Nutrition Inc.). See Supplementary Information for more diet details.

Dietary induction phase. After a one week acclimatization period on standard rat chow, body composition measurement was obtained and rats $(\mathrm{N}=80)$ were randomly assigned to either the HF diet $(45 \%$ of total cal from fat; OpenSource Diets product no. D12451; researchdiets.com; see Table 1 and Fig. S2; $\mathrm{N}=48$ rats) or the LF diet (10\% of total cal from fat; OpenSource Diets product no. D12450B; researchdiets.com; see Table 1 and Fig. S3; $\mathrm{N}=32$ rats), using an online randomizer (www.randomizer.org). The justification for the greater number of rats assigned to the HF diet was the anticipation that some subjects on this diet would not become obese $\mathrm{e}^{10}$. At the time of randomization to the operative procedure, there would need to be 30 rats in each diet group (see Fig. 1); so, the starting number of rats on the HF diet needed to be well over 30 . Rats were caged in pairs, and fed their assigned diet ad libitum for 6 months, which was defined as the dietary induction phase (Fig. 1).

Surgical procedure. Surgical procedures were based on a prior description ${ }^{10}$. After the dietary induction phase, body composition measurement was obtained, and within each group the 30 rats with the highest body weight were then randomly assigned to either massive or sham resection (Fig. 1), using the above online randomizer. Rats were fasted for $24 \mathrm{~h}$ prior to operation, but with free access to water. Anesthetic induction was obtained with $5 \%$ isoflurane via a nose cone and maintained with $2 \%$ isoflurane throughout the surgical procedure $^{10}$. After ensuring adequate anesthesia, subcutaneous cefovecin (20 mg; a long acting cephalosporin) was administered, the ventral hair was clipped, the abdomen was scrubbed with antiseptic solution (Povidone Iodine Topical Solution, 10\% USP, Allegiance, Cardinal Health), and the surgical field was isolated with sterile disposable drapes. A 6-cm midline laparotomy from the xiphoid process to urethral meatus was performed. The small intestine was exteriorized, and the entire length (from the pylorus to the cecum) was measured using a cotton umbilical tape. Intestinal surfaces were kept moist during the procedure with saline-soaked gauze.

Rats assigned to massive resection (Groups 2 and 4) underwent a 75\% distal enterectomy (Figs. S4-S6). The proximal transection line was in the mid jejunum, at the junction of the proximal one-quarter and distal three-quarters of the small intestinal length (Fig. S4). The distal transection line was in the distal ileum, $1 \mathrm{~cm}$ proximal to cecum (i.e., the ileocecal intersection was preserved). Mesenteric vessels were controlled using 4-0 silk (Fig. S5). An end-to-end jejunoileal anastomosis was performed using a single layer of running sutures with 6-0 silk (Ethicon Perma-Hand Silk, catalog no. K889H; see Fig. S6 for technical details). Rats assigned to sham resection (Groups 1 and 3) underwent a transection of the mid jejunum at the junction of the proximal one-quarter and distal three-quarters of the small intestinal length, without resection of any bowel (Fig. S4). The transected jejunum simply was re-connected with an end-to-end anastomosis as above.

The musculoaponeurotic layer of the abdominal wall was closed with a single running 3-0 polyester suture (Ethibond Excel, catalog no. X558H). Just prior to tying the polyester suture, $10 \mathrm{~mL}$ of saline was injected into the peritoneal cavity for postoperative hydration. The skin then was closed with staples (Autoclip $9 \mathrm{~mm}$ stainless steel clips; Mikron Precision, Inc., Catalog No. 427631). A one-time application of topical antibiotic ointment (Fura-Zone, $0.2 \%$ nitrofurazone; Squire Laboratories, Inc.) was spread along the skin staple line. An injection of long-acting buprenorphine ( $0.1 \mathrm{mg}$ subcutaneously) was given prior to recovery from anesthesia.

Postoperative observation phase. After the surgical procedure, all rats were fed ad libitum with an Intermediate-Fat (IF) diet (25\% of total cal from fat; OpenSource Diets Product No. D14041301; researchdiets. com; see Table 1 and Fig. S7). Body composition measurement was obtained at the end of the 60-day observation phase (Fig. 1). Euthanasia then was performed under deep isoflurane anesthesia with a laparotomy, bilateral diaphragm incision, and exsanguination by cardiotomy. The small intestine from the stomach to the cecum was resected en bloc, grossly inspected, measured for length, and photographed.

Body composition measurement. Body composition was determined noninvasively with quantitative magnetic resonance $(\mathrm{QMR})^{10}$ prior to the dietary induction phase, prior to the surgical procedure, and then prior to euthanasia, using an EchoMRI 700 Whole Body Composition Analyzer (Echo Medical Systems; echomri.com).

Statistical analysis. Data was analyzed using IBM SPSS Statistics for Windows, Version 22.0. Armonk, NY: IBM Corp. All results were expressed as mean \pm SD. ANOVA and t-testing were used to groups of data. Significance was defined as $p<0.05$. 


\begin{tabular}{|l|l|l|l|}
\hline Quantity & $\begin{array}{l}\text { High-fat diet } \\
(\mathbf{N = 4 8})\end{array}$ & $\begin{array}{l}\text { Low-fat diet } \\
(\mathbf{N}=32)\end{array}$ & $\boldsymbol{P}$ value $^{*}$ \\
\hline Body weight $(\mathrm{g})$ & $385 \pm 16$ & $389 \pm 20$ & 0.310 \\
\hline Body fat $(\mathrm{g})$ & $41.2 \pm 4.7$ & $42.0 \pm 5.7$ & 0.474 \\
\hline
\end{tabular}

Table 2. Body weight and fat prior to dietary induction. Values obtained just prior to 6-month dietary induction phase. Data $=$ mean $\pm \mathrm{SD} ;{ }^{*}$ unpaired t-test.

\begin{tabular}{|l|l|l|c|}
\hline Quantity & $\begin{array}{l}\text { High-fat diet } \\
(\mathbf{N}=30)\end{array}$ & $\begin{array}{l}\text { Low-fat diet } \\
(\mathbf{N}=30)\end{array}$ & $\boldsymbol{P}_{\text {value }^{*}}$ \\
\hline Body weight $(\mathrm{g})$ & $884 \pm 95$ & $741 \pm 75$ & $<0.0001$ \\
\hline Body fat (g) & $318 \pm 59$ & $186 \pm 45$ & $<0.0001$ \\
\hline Body fat (\%) & $35.8 \pm 3.9$ & $24.9 \pm 4.6$ & $<0.0001$ \\
\hline Lean weight (g) & $566 \pm 54$ & $555 \pm 53$ & 0.416 \\
\hline Lean weight (\%) & $64.2 \pm 3.9$ & $75.1 \pm 4.6$ & $<0.0001$ \\
\hline
\end{tabular}

Table 3. Preoperative body composition, high-fat $v s$. low-fat diet. Values obtained after 6-month dietary induction phase, immediately prior to resection. Data $=$ mean $\pm \mathrm{SD}$; ${ }^{*}$ unpaired $\mathrm{t}$-test.

\begin{tabular}{|l|l|l|l|}
\hline Group & Operated (N) & Died $(\mathbf{N})$ & Left for analysis (N) \\
\hline 1 (HFD, Sham) & 15 & 1 & 14 \\
\hline 2 (HFD, Massive) & 15 & 1 & 14 \\
\hline 3 (LFD, Sham) & 15 & 2 & 13 \\
\hline 4 (LFD, Massive) & 15 & 0 & 15 \\
\hline Sum & 60 & 4 & 56 \\
\hline
\end{tabular}

Table 4. Operative mortality. HFD = High-Fat Diet; $L F D=$ Low-Fat Diet; Sham = sham resection; Massive $=75 \%$ distal small intestinal resection.

\section{Results}

At the initiation of the study, the weight and body fat of the rats assigned to HFD $(\mathrm{N}=48)$ and LFD (N=32) just prior to the dietary induction phase were not different (Table 2 and Fig. S8). After the 6-month dietary induction phase, HFD and LFD rats were randomized to four intestinal resection groups $(1=$ HFD sham resection, $2=$ HFD massive resection, 3 =LFD sham resection, 4 =LFD massive resection; see Fig. S9A), following the design shown in Fig. 1. After completion of the 6-month dietary induction phase, the average weight, body fat, and percent body fat of HFD rats was greater than the LFD diet rats (Table 2); the former group gained an average of $\sim 500 \mathrm{~g}$ of weight and $\sim 277 \mathrm{~g}$ of fat, while the latter had respective gains of $\sim 352 \mathrm{~g}$ and $\sim 144 \mathrm{~g}$. The lean mass of the HFD vs. LFD rats was not different at the end of the 6-month dietary induction phase (Table 3 ).

Four rats died after the surgical procedure secondary to anastomotic leakage or intraabdominal sepsis $(6.7 \%$ operative mortality), leaving $\geq 13$ rats in each group for analysis (Table 4 ). The effect of diet and resection on total body weight (TBW) is shown in Table 5. Comparing all four treatment groups at single time points, there was no statistical difference in TBW among the four treatment groups neither prior to resection nor at two months after resection (i.e., at necropsy). Performing paired comparisons within each group across time (from the preoperative to the necropsy time point), there was a large (18\%) decrease in TBW in group 2 (HFD massive resection), with a marginal (2\%) decrease in TBW in group 1 (HFD sham resection). There was a modest (5\%) decrease in TBW in group 4 (LFD massive resection), with a modest (7\%) increase in TBW in group 3 (LFD sham resection). The decrease in TBW experienced by group 2 (HFD massive resection) was approximately four-fold greater than in group 4 (LFD massive resection). In the sham resection groups, there was a similar four-fold difference in TBW change between groups 1 and 3, favoring the latter (LFD sham resection). A flow diagram summarizing the fate of all 80 rats in this report is shown in Fig. S10.

The effect of diet and resection on body fat (BF) is shown in Table 6. Comparing all four treatment groups at a single time point, group 1 and 2 (HFD rats) had greater BF than groups 1 and 3 (LFD rats) at the preoperative time point. Performing paired comparisons within each group across time (from the preoperative to the necropsy time point), there was a large (45\%) decrease in BF in group 2 (HFD massive resection), with no significant decrease in BF in group 1 (HFD sham resection). There was a smaller (15\%) decrease in BF in group 4 (LFD massive resection), with a large (27\%) increase in BF in group 3 (LFD sham resection). The decrease in $\mathrm{BF}$ experienced by group 2 (HFD massive resection) was approximately five-fold greater than in group 4 (LFD massive resection). In the sham resection groups, there was a similar five-fold difference in BF change between groups 1 and 3, favoring the latter (LFD sham resection). 


\begin{tabular}{|c|c|c|c|c|c|c|}
\hline & \multicolumn{6}{|c|}{ Total body weight } \\
\hline & Pre-diet (g) & Preop (g) & Necrop (g) & $\begin{array}{l}\Delta \text { (Change), } \\
\text { Necrop - preop } \\
(\mathrm{g})\end{array}$ & $\begin{array}{l}\Delta \% \\
\text { (\% of Preop) }\end{array}$ & $\begin{array}{l}\text { Paired } t \text {-test } \\
\text { (Preop vs. Necrop) }\end{array}$ \\
\hline $\begin{array}{l}\text { Mean, Group } 1 \\
\text { (HFD Sham) }\end{array}$ & $379.4 \pm 18.6$ & $877.0 \pm 112.3$ & $858.5 \pm 104.7$ & $(18.5) \pm 36.0$ & $(1.9) \pm 4.0$ & 0.038 \\
\hline $\begin{array}{l}\text { Mean, Group } 2 \\
\text { (HFD Massive) }\end{array}$ & $388.5 \pm 17.3$ & $880.7 \pm 89.0$ & $717.6 \pm 97.4$ & $(163.1) \pm 80.1$ & $(18.4) \pm 8.4$ & $<0.0001$ \\
\hline $\begin{array}{l}\text { Mean, Group } 3 \\
\text { (LFD Sham) }\end{array}$ & $389.4 \pm 23.1$ & $738.4 \pm 75.2$ & $789.0 \pm 87.8$ & $50.7 \pm 27.6$ & $6.8 \pm 3.7$ & $<0.0001$ \\
\hline $\begin{array}{l}\text { Mean, Group } 4 \\
\text { (LFD Massive) }\end{array}$ & $383.2 \pm 14.5$ & $718.6 \pm 63.2$ & $681.7 \pm 69.5$ & $(36.9) \pm 29.5$ & $(5.2) \pm 4.1$ & 0.00013 \\
\hline $\begin{array}{l}\text { ANOVA, } \\
\text { Groups } 1-4\end{array}$ & 0.45 & 0.27 & 0.22 & $<0.0001$ & $<0.0001$ & \\
\hline \multicolumn{7}{|l|}{ Unpaired t-test } \\
\hline $1 \mathrm{v.} 2$ & & & & $<0.0001$ & $<0.0001$ & \\
\hline 3 v. 4 & & & & $<0.0001$ & $<0.0001$ & \\
\hline 1 v. 3 & & & & $<0.0001$ & $<0.0001$ & \\
\hline 2 v. 4 & & & & $<0.0001$ & $<0.0001$ & \\
\hline
\end{tabular}

Table 5. Effect of diet and resection on total body weight. Values shown are mean \pm standard deviation. Red values in parentheses (xx.x) represent a negative change (decrease). Pre-Diet = prior to the 6-mo dietary induction period; Preop = Preoperative (after dietary induction, immediately prior to resection); Necropsy = immediately prior to necropsy (two months after resection); HFD = High-Fat Diet; LFD = Low-Fat Diet; Massive $=$ Massive $75 \%$ distal small intestinal resection; Sham $=$ Sham resection .

\begin{tabular}{|c|c|c|c|c|c|c|c|c|}
\hline & \multicolumn{5}{|c|}{ Body Fat Mass } & \multicolumn{3}{|c|}{ Body Fat Mass as percent of TBW } \\
\hline & Preop (g) & Necrop (g) & $\begin{array}{l}\Delta \text { (Change), } \\
\text { Necrop - } \\
\text { preop } \\
(\mathrm{g})\end{array}$ & $\begin{array}{l}\Delta \% \\
\text { (\% of Preop) }\end{array}$ & $\begin{array}{l}\text { Paired t-test } \\
\text { (Preop vs. } \\
\text { Necrop) }\end{array}$ & $\begin{array}{l}\text { Preop } \\
\text { \%Body fat }\end{array}$ & $\begin{array}{l}\text { Necrop } \\
\text { \%Body fat }\end{array}$ & $\begin{array}{l}\text { Paired t-test } \\
\text { (Preop v. } \\
\text { Necrop) }\end{array}$ \\
\hline $\begin{array}{l}\text { Mean, Group } 1 \\
\text { (HFD Sham) }\end{array}$ & $315.8 \pm 69.3$ & $306.7 \pm 62.6$ & $(9.1) \pm 31.2$ & $(1.9) \pm 9.4$ & 0.15 & $35.7 \pm 4.8$ & $35.5 \pm 4.5$ & 0.39 \\
\hline $\begin{array}{l}\text { Mean, Group } 2 \\
\text { (HFD Mas- } \\
\text { sive) }\end{array}$ & $315.6 \pm 50.4$ & $177.1 \pm 61.4$ & $(138.5) \pm 38.2$ & $(44.9) \pm 13.7$ & $<0.0001$ & $35.7 \pm 3.4$ & $24.2 \pm 6.0$ & $<0.0001$ \\
\hline $\begin{array}{l}\text { Mean, Group } 3 \\
\text { (LFD Sham) }\end{array}$ & $189.8 \pm 47.9$ & $239.9 \pm 56.1$ & $50.1 \pm 19.0$ & $26.9 \pm 10.3$ & $<0.0001$ & $25.5 \pm 4.7$ & $30.1 \pm 5.1$ & $<0.0001$ \\
\hline $\begin{array}{l}\text { Mean, Group } 4 \\
\text { (LFD Massive) }\end{array}$ & $180.8 \pm 43.2$ & $155.6 \pm 48.4$ & $(25.2) \pm 26.5$ & $(14.7) \pm 15.4$ & 0.0012 & $25.0 \pm 4.9$ & $22.6 \pm 5.8$ & 0.0066 \\
\hline $\begin{array}{l}\text { ANOVA, } \\
\text { Groups } 1-4\end{array}$ & $<0.0001$ & $<0.0001$ & $<0.0001$ & $<0.0001$ & & $<0.0001$ & $<0.0001$ & \\
\hline \multicolumn{9}{|l|}{$\begin{array}{l}\text { Unpaired } \\
\text { t-test }\end{array}$} \\
\hline 1 v. 2 & 0.50 & $<0.0001$ & $<0.0001$ & $<0.0001$ & & 0.5 & $<0.0001$ & \\
\hline 3 v. 4 & 0.30 & 0.00011 & $<0.0001$ & $<0.0001$ & & 0.41 & 0.00066 & \\
\hline 1 v. 3 & $<0.0001$ & 0.0037 & $<0.0001$ & $<0.0001$ & & $<0.0001$ & 0.0036 & \\
\hline 2 v. 4 & $<0.0001$ & 0.15 & $<0.0001$ & $<0.0001$ & & $<0.0001$ & 0.24 & \\
\hline
\end{tabular}

Table 6. Effect of diet and resection on body fat. Values shown are mean \pm standard deviation. Red values in parentheses (xx.x) represent a negative change (decrease). Preop = Preoperative (after dietary induction, immediately prior to resection); Necropsy=immediately prior to necropsy (two months after resection); $\mathrm{HFD}=$ High-Fat Diet; LFD = Low-Fat Diet; Massive = Massive 75\% distal small intestinal resection; Sham $=$ Sham resection; $\mathrm{TBW}=$ Total Body Weight.

The effect of diet and resection on lean mass (LM) is shown in Table 7. Comparing all four treatment groups at single time points, there was no statistical difference in LM among the four treatment groups neither prior to resection nor at two months after resection (i.e., at necropsy). Performing paired comparisons within each group across time (from the preoperative to the necropsy time point), there was a modest (4\%) decrease in LM in group 2 (HFD massive resection), with no statistical change in LM in group 1 (HFD sham resection). There also was a modest (3\%) decrease in LM in group 4 (LFD massive resection), with no statistical change in LM in group 3 (LFD sham resection). Of note, the change in LM from the preoperative to the necropsy time point between group 2 (HFD massive resection) vs. group 4 (LF massive resection) was not statistically different. 


\begin{tabular}{|c|c|c|c|c|c|c|c|c|}
\hline & \multicolumn{5}{|c|}{ Body Lean Mass } & \multicolumn{3}{|c|}{ Lean Mass as percent of TBW } \\
\hline & Preop (g) & Necrop $(g)$ & $\begin{array}{l}\Delta \text { (Change), } \\
\text { Necrop - } \\
\text { Preop } \\
(\mathrm{g})\end{array}$ & $\begin{array}{l}\Delta \% \\
\text { (\% of Preop) }\end{array}$ & $\begin{array}{l}\text { Paired t-test } \\
\text { (Preop vs. } \\
\text { Necrop) }\end{array}$ & $\begin{array}{l}\text { Preop } \\
\text { \%Lean Mass }\end{array}$ & $\begin{array}{l}\text { Necrop } \\
\text { \%Lean Mass }\end{array}$ & $\begin{array}{l}\text { Paired t-test } \\
\text { (Preop v. } \\
\text { Necrop) }\end{array}$ \\
\hline $\begin{array}{l}\text { Mean, } \\
\text { Group } 1 \\
\text { (HFD Sham) }\end{array}$ & $482.8 \pm 47.3$ & $480.6 \pm 48.9$ & $(2.1) \pm 18.0$ & $(0.4) \pm 3.8$ & 0.33 & $55.4 \pm 4.0$ & $56.3 \pm 4.5$ & 0.080 \\
\hline $\begin{array}{l}\text { Mean, } \\
\text { Group } 2 \\
\text { (HFD Mas- } \\
\text { sive) }\end{array}$ & $493.1 \pm 35.2$ & $472.7 \pm 41.3$ & $(20.5) \pm 14.5$ & $(4.2) \pm 3.0$ & $<0.0001$ & $56.3 \pm 5.0$ & $66.5 \pm 6.2$ & $<0.0001$ \\
\hline $\begin{array}{l}\text { Mean, } \\
\text { Group } 3 \\
\text { (LFD Sham) }\end{array}$ & $479.8 \pm 41.7$ & $480.8 \pm 44.8$ & $1.0 \pm 11.3$ & $0.2 \pm 2.3$ & 0.37 & $65.2 \pm 4.1$ & $61.2 \pm 4.7$ & $<0.0001$ \\
\hline $\begin{array}{l}\text { Mean, } \\
\text { Group } 4 \\
\text { (LFD Mas- } \\
\text { sive) }\end{array}$ & $482.0 \pm 42.8$ & $466.0 \pm 47.6$ & $(16.0) \pm 12.6$ & $(3.4) \pm 2.8$ & 0.00011 & $67.2 \pm 4.2$ & $68.5 \pm 5.5$ & 0.075 \\
\hline $\begin{array}{l}\text { ANOVA, } \\
\text { Groups } 1-4\end{array}$ & 0.84 & 0.79 & 0.0004 & 0.0004 & & $<0.0001$ & $<0.0001$ & \\
\hline \multicolumn{9}{|l|}{$\begin{array}{l}\text { Unpaired } \\
\text { t-test }\end{array}$} \\
\hline 1 v. 2 & & & 0.0032 & 0.0032 & & 0.29 & $<0.0001$ & \\
\hline 3 v. 4 & & & 0.00047 & 0.00053 & & 0.11 & 0.00046 & \\
\hline 1 v. 3 & & & 0.30 & 0.32 & & $<0.0001$ & 0.0046 & \\
\hline 2 v. 4 & & & 0.19 & 0.22 & & $<0.0001$ & 0.18 & \\
\hline
\end{tabular}

Table 7. Effect of diet and resection on lean mass. Values shown are mean \pm standard deviation. Red values in parentheses (xx.x) represent a negative change (decrease). Preop=Preoperative (after dietary induction, immediately prior to resection); Necropsy = immediately prior to necropsy (two months after resection); HFD = High-Fat Diet; $\mathrm{LFD}=$ Low-Fat Diet; Massive $=$ Massive $75 \%$ distal small intestinal resection; Sham $=$ Sham resection; TBW $=$ Total Body Weight.

\section{Discussion}

This study was a second test of the hypothesis that pre-existing obesity would preserve lean mass in a rat model of massive intestinal resection (i.e., surgically-induced SBS). The first test of this hypothesis was published in $2015^{10}$, and utilized a $50 \%$ small intestinal resection with a postoperative observation period of one month. This prior study did not convincingly demonstrate a protective effect of obesity; interestingly, no differential effect of $50 \%$ enterectomy vs. sham resection on body composition was demonstrable in this 2015 study $^{10}$. So in the second test of the above hypothesis (i.e., the present report), the size of the intestinal resection and the length of the postoperative observation period were increased to $75 \%$ and two months, respectively. The expectation for these changes in study design was that any protective effect of obesity on lean mass in this revised model would be more pronounced and possibly significant.

The data in the present report did not prove the hypothesis either; pre-existing obesity in a rat model of $75 \%$ distal small intestinal resection was not protective of lean mass two months after resection. Secondary conclusions that could be drawn from the data include: (1) the high-fat diet induced obesity (36\% body fat); (2) the low-fat diet resulted in marginal obesity ( $25 \%$ body fat); (3) massive resection produced substantially greater weight loss than sham resection for rats on either diet; and (4) rats pre-conditioned with the high-fat diet lost more weight and body fat after massive resection compared to the low-fat diet rats. Of these secondary conclusions, numbers 1, 3, and 4 were expected outcomes, and support the validity of the study. In particular, the present study (which utilized a 75\% enterectomy) demonstrated a large effect of resection on body composition in comparison to sham resection; the prior 2015 study (which utilized a 50\% enterectomy), was unable to demonstrate such a differential effect ${ }^{10}$.

Regarding secondary conclusion number 2 , the low-fat diet rats in the present study had greater percent body fat $(25 \%)$ compared to the $18 \%$ body fat of similarly-preconditioned rats from the 2015 study (same rat strain) $)^{10}$. It is not clear why the low-fat diet rats in the present study attained this relatively high preoperative percent body fat, or whether these rats were "lean enough" to qualify as a "non-obese" comparator group for the rats on the high-fat diet. Experimental obesity in rats has been defined as a percent body fat of $20-25 \%{ }^{15-17}$, so the "non-obese" rats in the present study were on the margin of this definition. It is interesting to note, however, that low-fat diet rats in the present study actually gained weight and body fat after sham resection, while the high-fat diet rats did not; this result suggests a difference in the underlying response to stress between rats on these two diets. The relationship of this observation to the central hypothesis, which focused on lean mass, is not obvious.

The data from the present study do not support the "obesity paradox", in which better outcomes have been hypothesized for obese patients compared to non-obese patients suffering from a variety of diseases or conditions $^{18-25}$. From this perspective, the present report could be classified as a "negative" study. Possible causes for the finding of no support for the obesity paradox include: (1) the non-obese rats were not "lean enough" to 
be a comparator group (see above discussion); (2) the rats were too old about one year) at the study's conclusion to mount a vigorous response to surgical stress; (3) the rat may not adequately reproduce human physiology in this area, i.e., the rat may have been inadequate model; (4) the present study did not utilize physical fitness as a variable, which may be a relevant parameter in the obesity paradox ${ }^{25}$; or (5) the wrong endpoint (lean mass) was chosen for this study. Alternatively, this study may be clinically accurate in that the obesity paradox may not apply to massive intestinal resection and/or SBS.

A drawback of this study is that all of the rat subjects were male. The NIH has mandated that subject sex should be considered as a biological variable, and that grant applicants should balance male and female subjects in their preclinical investigations ${ }^{26}$. Of note, the study was designed and executed prior to the NIH's announcement of its intent to focus on sex as a biological variable. Regardless of that, the present study cannot discriminate any influence of subject sex on outcome, because there were no female subjects in this study.

Regarding the selection of an appropriate primary endpoint, in clinical cases of massive intestinal resection or SBS, relevant patient outcomes include survival, operative morbidity, nutritional parameters (including lean mass), long-term dependence on parenteral nutrition, and quality of life indicators. We selected lean mass as the best and most practical endpoint that was available to us for the rat model; this endpoint has been commonly employed in other studies on nutrition and metabolism in rats ${ }^{27-31}$. In addition to the evaluation of lean mass as the primary endpoint, there are secondary endpoints that could have been evaluated in the present study, including food intake, microbiome, intestinal adaptation, immunohistochemical staining, serum peptide levels, and other nutrition-relevant serum tests; of note, most of these secondary endpoints were examined in the 2015 study $^{10}$. We elected not to perform these analyses in the current study, because: (1) the relevance of these secondary endpoint data would not be clear, as the study was negative with respect to the primary endpoint; and (2) we did not want to expend resources in the pursuit of costly secondary endpoint data which would have doubtful relevance.

In regard to analysis of secondary endpoints (not done in this study), the relationship between post-resection body composition and intestinal adaptation, as an example, would be a relevant research topic. However, the study herein was not designed to interrogate that relationship. Going back into this study to examine the body composition-adaptation relationship or other secondary endpoints would essentially be a post-hoc analysis of a negative study, with the hope of generating a statistically significant finding. Because our study failed to find a difference in its original, intended mission, any statistical findings in a post-hoc analysis would have questionable relevance.

The use of post-hoc analysis is controversial and should be utilized with caution, if used at all ${ }^{32-34}$. In addition, given the variability in our data, we do not believe our study was statistically powered to perform a post-hoc analysis of the body composition-adaption relationship. That is, using an $\mathrm{N} \leq 15$ rats per group in this circumstance would risk a type II error (false negative). Instead of performing such a post-hoc analysis, it would be better to design a new properly-powered study, set up to specifically interrogate the relationship between post-resection body composition and intestinal adaptation (or other endpoints) in our rat model of $75 \%$ intestinal resection.

In summary, this was a report of a second study in which the effect of pre-resection obesity on preservation of lean body mass after massive intestinal resection was tested in rats. In this second study, a longer postoperative observation period and a greater intestinal resection was utilized compared to the first study. Neither study was able to demonstrate a protective effect of obesity on lean mass after massive intestinal resection.

Received: 11 November 2020; Accepted: 3 June 2021

Published online: 21 June 2021

\section{References}

1. Howard, L., Ament, M., Fleming, C. R., Shike, M. \& Steiger, E. Current use and clinical outcome of home parenteral and enteral nutrition therapies in the United States. Gastroenterology 109(2), 355-365 (1995).

2. DiBaise JK, Rees Parrish C, Thompson JS. Short Bowel Syndrome: Practical Approach to Management: CRC Press; 2017.

3. Kurlberg, G., Forssell, H. \& Aly, A. National registry of patients with short bowel syndrome. Transpl. Proc. 36(2), 253-254. https:// doi.org/10.1016/j.transproceed.2003.12.021 (2004).

4. Scolapio, J. S. \& Fleming, C. R. Short bowel syndrome. Gastroenterol Clin N Am. 27(2), 467-479 (1998).

5. Thompson, J. S., DiBaise, J. K., Iyer, K. R., Yeats, M. \& Sudan, D. L. Postoperative short bowel syndrome. J Am Coll Surg. 201(1), 85-89. https://doi.org/10.1016/j.jamcollsurg.2005.02.034 (2005).

6. Welters, C. F., Dejong, C. H., Deutz, N. E. \& Heineman, E. Intestinal adaptation in short bowel syndrome. ANZ J Surg. 72(3), 229-236 (2002).

7. Crenn, P. et al. Net digestive absorption and adaptive hyperphagia in adult short bowel patients. Gut 53(9), 1279-1286 (2004).

8. Thompson, J. S. et al. Pre-resection gastric bypass reduces post-resection body mass index but not liver disease in short bowel syndrome. Am J Surg. 207(6), 942-948. https://doi.org/10.1016/j.amjsurg.2013.10.019 (2014).

9. Thompson JS, Weseman R, Rochling F, Grant W, Botha J, Mercer DF, editors. Pre-resection BMI influences post resection BMI in short bowel syndrome. XI International Small Bowel Transplant Symposium; 2009; Bologna, Italy: Medimond International Proceedings/ Monduzzi Editore.

10. Yanala, U. R., Reidelberger, R. D., Thompson, J. S., Shostrom, V. K. \& Carlson, M. A. Effect of proximal versus distal 50\% enterectomy on nutritional parameters in rats preconditioned with a high-fat diet or regular chow. Sci Rep. 5, 17331. https://doi.org/10. 1038/srep17331 (2015).

11. Committee for the Update of the Guide for the Care and Use of Laboratory Animals. Guide for the Care and Use of Laboratory Animals: Washington, DC: The National Academies Press; 2011.

12. American Veterinary Medical Association Panel on Euthanasia. AVMA Guidelines for the Euthanasia of Animals: 2013 EditionSchaumberg, IL: American Veterinary Medical Association; 2013.

13. Kilkenny C, Browne WJ, Cuthill IC, Emerson M, Altman DG. Improving bioscience research reporting: the ARRIVE guidelines for reporting animal research. PLoS Biol. 2010;8(6):e1000412. 
14. Percie du Sert N, Ahluwalia A, Alam S, Avey MT, Baker M, Browne WJ, Clark A, Cuthill IC, Dirnagl U, Emerson M, Garner P, Holgate ST, Howells DW, Hurst V, Karp NA, Lazic SE, Lidster K, MacCallum CJ, Macleod M, Pearl EJ, Petersen OH, Rawle F, Reynolds P, Rooney K, Sena ES, Silberberg SD, Steckler T, Wurbel H. Reporting animal research: Explanation and elaboration for the ARRIVE guidelines 2.0. PLoS Biol. 2020;18(7):e3000411. DOI: https://doi.org/10.1371/journal.pbio.3000411

15. Bueter, M. et al. Vagal sparing surgical technique but not stoma size affects body weight loss in rodent model of gastric bypass. Obes Surg. 20(5), 616-622. https://doi.org/10.1007/s11695-010-0075-5 (2010).

16. Reidelberger, R. et al. Effects of leptin replacement alone and with exendin-4 on food intake and weight regain in weight-reduced diet-induced obese rats. Am J Physiol. 302(12), E1576-1585. https://doi.org/10.1152/ajpendo.00058.2012 (2012).

17. Trevaskis, J. L. et al. Amylin-mediated restoration of leptin responsiveness in diet-induced obesity: magnitude and mechanisms. Endocrinology 149(11), 5679-5687. https://doi.org/10.1210/en.2008-0770 (2008).

18. Gonzalez, M. C., Pastore, C. A., Orlandi, S. P. \& Heymsfield, S. B. Obesity paradox in cancer: new insights provided by body composition. Am J Clin Nutr. 99(5), 999-1005 (2014).

19. Lavie, C. J., Milani, R. V. \& Ventura, H. O. Obesity and cardiovascular diseaserisk factor, paradox, and impact of weight loss. J Am Coll Cardiol. 53(21), 1925-1932 (2009).

20. Mullen, J. T. et al. Impact of body mass index on perioperative outcomes in patients undergoing major intra-abdominal cancer surgery. Ann Surg Oncol. 15(8), 2164-2172 (2008).

21. Mullen, J. T., Moorman, D. W. \& Davenport, D. L. The obesity paradox: body mass index and outcomes in patients undergoing nonbariatric general surgery. Ann Surg. 250(1), 166-172 (2009).

22. Oliveros, H. \& Villamor, E. Obesity and mortality in critically Ill adults: a systematic review and meta-analysis. Obesity 16(3), 515-521 (2008).

23. Oreopoulos, A., Kalantar-Zadeh, K., Sharma, A. M. \& Fonarow, G. C. The obesity paradox in the elderly: potential mechanisms and clinical implications. Clinics Geriatric Med. 25(4), 643-659 (2009).

24. Lennon, H., Sperrin, M., Badrick, E. \& Renehan, A. G. The obesity paradox in cancer: a review. Curr Oncol Rep. 18(9), 56. https:// doi.org/10.1007/s11912-016-0539-4 (2016).

25. Elagizi, A. et al. An overview and update on obesity and the obesity paradox in cardiovascular diseases. Prog Cardiovasc Dis. 61(2), 142-150. https://doi.org/10.1016/j.pcad.2018.07.003 (2018).

26. Arnegard, M. E., Whitten, L. A., Hunter, C. \& Clayton, J. A. Sex as a biological variable: a 5-year progress report and call to action. J Womens Health (Larchmt). 29(6), 858-864. https://doi.org/10.1089/jwh.2019.8247 (2020).

27. Gillingham, M. B., Dahly, E. M., Murali, S. G. \& Ney, D. M. IGF-I treatment facilitates transition from parenteral to enteral nutrition in rats with short bowel syndrome. Am J Physiol Regul Integr Comp Physiol. 284(2), R363-371. https://doi.org/10.1152/ajpre gu.00247.2002 (2003).

28. Adam, C. L., Williams, P. A., Garden, K. E., Thomson, L. M. \& Ross, A. W. Dose-dependent effects of a soluble dietary fibre (pectin) on food intake, adiposity, gut hypertrophy and gut satiety hormone secretion in rats. PLoS ONE 10(1), e0115438. https://doi.org/ 10.1371/journal.pone.0115438 (2015).

29. Bertin, E., Ruiz, J. C., Mourot, J., Peiniau, P. \& Portha, B. Evaluation of dual-energy X-ray absorptiometry for body-composition assessment in rats. J Nutr. 128(9), 1550-1554. https://doi.org/10.1093/jn/128.9.1550 (1998).

30. Mika, A. et al. Exercise is more effective at altering gut microbial composition and producing stable changes in lean mass in juvenile versus adult male F344 Rats. PLoS ONE 10(5), e0125889. https://doi.org/10.1371/journal.pone.0125889 (2015).

31. Yu, B. P., Masoro, E. J., Murata, I., Bertrand, H. A. \& Lynd, F. T. Life span study of SPF Fischer 344 male rats fed ad libitum or restricted diets: longevity, growth, lean body mass and disease. J Gerontol. 37(2), 130-141. https://doi.org/10.1093/geronj/37.2. 130 (1982).

32. Levine, M. \& Ensom, M. H. Post hoc power analysis: an idea whose time has passed?. Pharmacotherapy 21(4), 405-409. https:// doi.org/10.1592/phco.21.5.405.34503 (2001).

33. Curran-Everett, D. \& Milgrom, H. Post-hoc data analysis: benefits and limitations. Curr Opin Allergy Clin Immunol. 13(3), $223-224$. https://doi.org/10.1097/ACI.0b013e3283609831 (2013).

34. Shanks, D. R. Regressive research: The pitfalls of post hoc data selection in the study of unconscious mental processes. Psychon Bull Rev. 24(3), 752-775. https://doi.org/10.3758/s13423-016-1170-y (2017).

\section{Acknowledgements}

This study is the result of work supported in part with resources and the use of facilities at the Omaha VA Medical Center (Nebraska-Western Iowa Health Care System). The authors would like to acknowledge the technical assistance of Gerri Siford and Chris Hansen. This work was funded through the Department of Surgery at the University of Nebraska Medical Center.

\section{Author contributions}

NSP and MAC wrote the main manuscript text and prepared prepared the Figures and Tables. All authors reviewed the manuscript.

\section{Competing interests}

The authors declare no competing interests.

\section{Additional information}

Supplementary Information The online version contains supplementary material available at https://doi.org/ 10.1038/s41598-021-92510-7.

Correspondence and requests for materials should be addressed to M.A.C.

Reprints and permissions information is available at www.nature.com/reprints.

Publisher's note Springer Nature remains neutral with regard to jurisdictional claims in published maps and institutional affiliations. 
(c) (i) Open Access This article is licensed under a Creative Commons Attribution 4.0 International cc) License, which permits use, sharing, adaptation, distribution and reproduction in any medium or format, as long as you give appropriate credit to the original author(s) and the source, provide a link to the Creative Commons licence, and indicate if changes were made. The images or other third party material in this article are included in the article's Creative Commons licence, unless indicated otherwise in a credit line to the material. If material is not included in the article's Creative Commons licence and your intended use is not permitted by statutory regulation or exceeds the permitted use, you will need to obtain permission directly from the copyright holder. To view a copy of this licence, visit http://creativecommons.org/licenses/by/4.0/.

This is a U.S. Government work and not under copyright protection in the US; foreign copyright protection may apply 2021 